\title{
Effectiveness of seasonal influenza vaccination during pregnancy in preventing influenza infection in infants, England, 2013/14
}

G Dabrera ${ }^{1,2,3}$, H Zhao 3 , N Andrews³, F Begum³ ${ }^{3}$ H K Green ${ }^{3}$, J Ellis ${ }^{4}$, K Elias ${ }^{5}$, M Donati ${ }^{6}$, M Zambon $^{4}$,

R Pebody (Richard.Pebody@phe.gov.uk) ${ }^{3}$

1. Field Epidemiology Training Programme, Public Health England, London, United Kingdom

2. European Programme for Intervention Epidemiology Training (EPIET), European Centre for Disease Prevention and Control (ECDC), Stockholm, Sweden

3. Centre for Infectious Disease Surveillance and Control, Public Health England, London, United Kingdom

4. Virus Reference Department, Microbiology Services, Public Health England, London, United Kingdom

5. Coach House Surgery, Watford, United Kingdom

6. Public Health England Bristol Public Health Laboratory, Bristol, United Kingdom

Citation style for this article:

Citation style for this article:

pregnancy in preventing influenza infection in infants, England, 2013/14. Euro Surveill. 2014;19(45): pii=20959. Available online: http://www.eurosurveillance.org/ pregnancy in preventing influenza infe
ViewArticle.aspx?Articleld $=20959$

Article submitted on 04 November 2014 / published on 13 November 2014

In this study we used the screening method to estimate the effectiveness of seasonal influenza vaccination during pregnancy in preventing influenza virus infection and influenza-related hospitalisation in infants undersix months, in England in the 2013/14 season. Seasonal influenza vaccination in pregnancy was $71 \%$ (95\% Cl: $24-89 \%$ ) effective in preventing infant influenza virus infection and $64 \%$ (95\% Cl: 6-86\%) effective in preventing infant influenza hospitalisation, and should be recommended in pregnancy.

Our study assessed the effectiveness of seasonal influenza vaccination during pregnancy in preventing influenza virus infection and influenza-related hospitalisation in infants under six months of age, in England in the influenza season $2013 / 14$. This study is the first to use the screening method to calculate such an estimate for preventing infant influenza virus infection.

\section{Background}

Influenza in infants aged under six months is responsible for a significant burden of illness, impacting on a range of health services. When comparing the incidence of cardiopulmonary-related hospitalisations during influenza seasons to the rest of the year, the average annual increase in the United States (US) was highest for children aged under six months (104 hospitalisations/10,000 children), compared to children aged between six months and 12 months and children aged between one year and three years (50/10,000 and 19/10,000 respectively) [1].

Similar age-specific hospitalisation trends have been observed in England [2]; additionally, higher rates of influenza-related paediatric intensive care unit admissions occurred among children agedunder one year $(0.7 / 100,000)$ compared to those aged from one to four years $(0.2 / 100,000)$ and five to nine years $(0.5 / 100,000)$ during the 2009 influenza $A\left(\mathrm{H}_{1} \mathrm{~N}_{1}\right)$ pandemic [3]. There are also an estimated 22.3 influenza-attributable consultations in primary care for every child aged under six months admitted to hospital, indicating the intense demands on primary care services [2]. Preventing influenza in those aged under six months is therefore an important health priority. These children are too young to receive the current seasonal influenza vaccines, which are only licensed in older children [4].

Influenza vaccination during pregnancy directly protects newborn infants from influenza virus infection through transplacental transfer of maternal antibodies [5]. Several countries including the US, Canada, the UK (UK) and other European countries, recommend seasonal influenza vaccination during pregnancy, mainly to protect pregnant women who are at increased risk of severe infection, as observed with 2009 pandemic influenza $A\left(\mathrm{H}_{1} \mathrm{~N}_{1}\right)[4,6,7]$. While few studies in other countries have examined the effectiveness of vaccination during pregnancy in preventing infant influenza infection [8-10], there has been no previous assessment of this in England, since its introduction in 2009.

\section{Data collection}

We defined a case as an infant aged under six months, born between 1 September 2013 and 31 January 2014, with laboratory-confirmed influenza infection by RT-PCR. Cases were retrospectively identified between 30 September 2013 and 18 May 2014 (the national seasonal influenza surveillance period), from the Respiratory DataMart System (RDMS), a sentinel laboratory surveillance system which collects influenza testing data predominantly from secondary care settings in England [11]. Cases were restricted to those born between 1 September 2013 and 31 January 2014 
which corresponds to the roll-out of the $2013 / 14$ programme of influenza vaccination during pregnancy.

Cases' general practitioners were sent postal questionnaires to identify if infants were hospitalised, if each infant's mother had received influenza vaccination during pregnancy (proportion of 'cases' vaccinated, PCV) and if so, the vaccination date. Up to three postal/fax reminders were sent to general practitioners in case of non-response.

A mother was classified as fully vaccinated in pregnancy if vaccination occurred at least 14 days before the infant's birth, (considered the minimum time for the mother to develop a full immune response) or unvaccinated if mothers were vaccinated after birth or not at all. Infants whose mothers were vaccinated less than 14 days before birth or had an unknown vaccination status or vaccination date, were excluded from analysis as the mothers' immune status was uncertain.

Seasonal influenza vaccination coverage for the population of pregnant women (PPV) in England was identified through a national electronic reporting system (ImmForm) which is used nationally by general practices in England to report vaccine administration for seasonal influenza vaccination. The end of season collection reported a $99.8 \%$ response rate in 2013/14 [12]. ImmForm data were used to identify the number of pregnant women (at any stage of pregnancy) registered in primary care, and the cumulative number of these women who received seasonal influenza vaccination between 1 September 2013 and 31 January 2014, at monthly intervals from end-October. Sub-national coverage was calculated for four regions of England (London, South, Midlands and North).

\section{Data analysis}

Data analysis was undertaken using Microsoft Excel 2007 (Microsoft, Redmond, Washington) and Stata version 12.0 (StataCorp, College Station, Texas). Characteristics of cases included in the analysis were reported, including age group, sex, influenza sub-type, hospitalisation status and maternal vaccination status.

We used the screening method to estimate vaccine effectiveness (VE); this approach has been used previously to estimate VE against influenza for other groups [13-15].

Crude VE was estimated separately for all influenza cases and for hospitalised influenza cases as:

$\mathrm{VE}=1-\left(\frac{P C V}{(1-P C V)} \times \frac{(1-P P V)}{P P V}\right)$

Adjusted VE for all laboratory-confirmed influenza infection and hospitalised influenza cases was estimated by using the natural logarithm of PPV in each region and month of birth as an offset in a logistic regression model where the outcome was vaccination status during pregnancy for the mother of each case, therefore allowing for individually matched coverage for each case by region and month of birth. Month of birth and region were included as potential confounders as these were both related to vaccine coverage and influenza activity during the influenza season.

\section{Results}

There were 43 infants with laboratory-confirmed influenza infection reported through RDMS, born between 1 September 2013 and 31 January 2014 (Figure).

Of these 43 cases, 37 were included in the analysis. Six cases were excluded: two with no response from general practitioners, one with unknown vaccination status of the mother in the returned questionnaire, two with unknown date of maternal vaccination and one with maternal vaccination less than 14 days before birth. Of these cases, 22 were male. Median age of infants at time of influenza testing date was 13 weeks (range 2-21 weeks). Twenty-two cases tested positive for 2009 pandemic influenza $A\left(\mathrm{H}_{1} \mathrm{~N}_{1}\right)$, nine for $A\left(\mathrm{H}_{3} \mathrm{~N}_{2}\right)$, five for influenza $A$ (unspecified) and one for influenza B.

Nationally, of 659,223 pregnant women, 262,081 $(39.8 \%)$ were reported to have received seasonal influenza vaccination in pregnancy in 2013/14. Five cases' mothers were reported to have received seasonal influenza vaccination in pregnancy. The median interval between maternal vaccination and birth was six weeks (range 4-12 weeks). The crude VE for preventing all influenza cases was estimated as $76 \%$ ( $95 \%$ confidence interval ( $\mathrm{Cl}): 39-93 \%)$ and the adjusted VE was $71 \%$ (95\% Cl: $24-89 \%)$.

Hospitalisation data were available for 36 cases; of these, 32 cases were hospitalised, including the same five cases as before. The crude VE for hospitalised influenza cases was $72 \%(95 \% \mathrm{Cl}$ : $26-92 \%)$; the adjusted VE was $64 \%$ (95\% Cl: 6-86\%).

\section{Discussion}

Our results demonstrate that vaccination during pregnancy effectively prevents laboratory-confirmed influenza infection and associated hospitalisation in infants in the first six months of life. These results are comparable with a previous randomised control trial (RCT) in Bangladesh showing that vaccination during pregnancy was $63 \%$ effective in preventing influenza in infants aged under six months [8]. Additionally, studies in the US have identified a VE of $48-91 \%$ in preventing influenza-related infant hospitalisations $[9,10]$. Our findings supplement the existing evidence for this intervention, underlining that previous results in earlier seasons in other settings are applicable to the UK and could have implications for other European countries also. Our VE estimate reflects a combination of both the direct effect of transplacental antibody 
Recruitment of cases included in the study on effectiveness of seasonal influenza vaccination in pregnancy in preventing influenza infection in infants, England, 2013/14

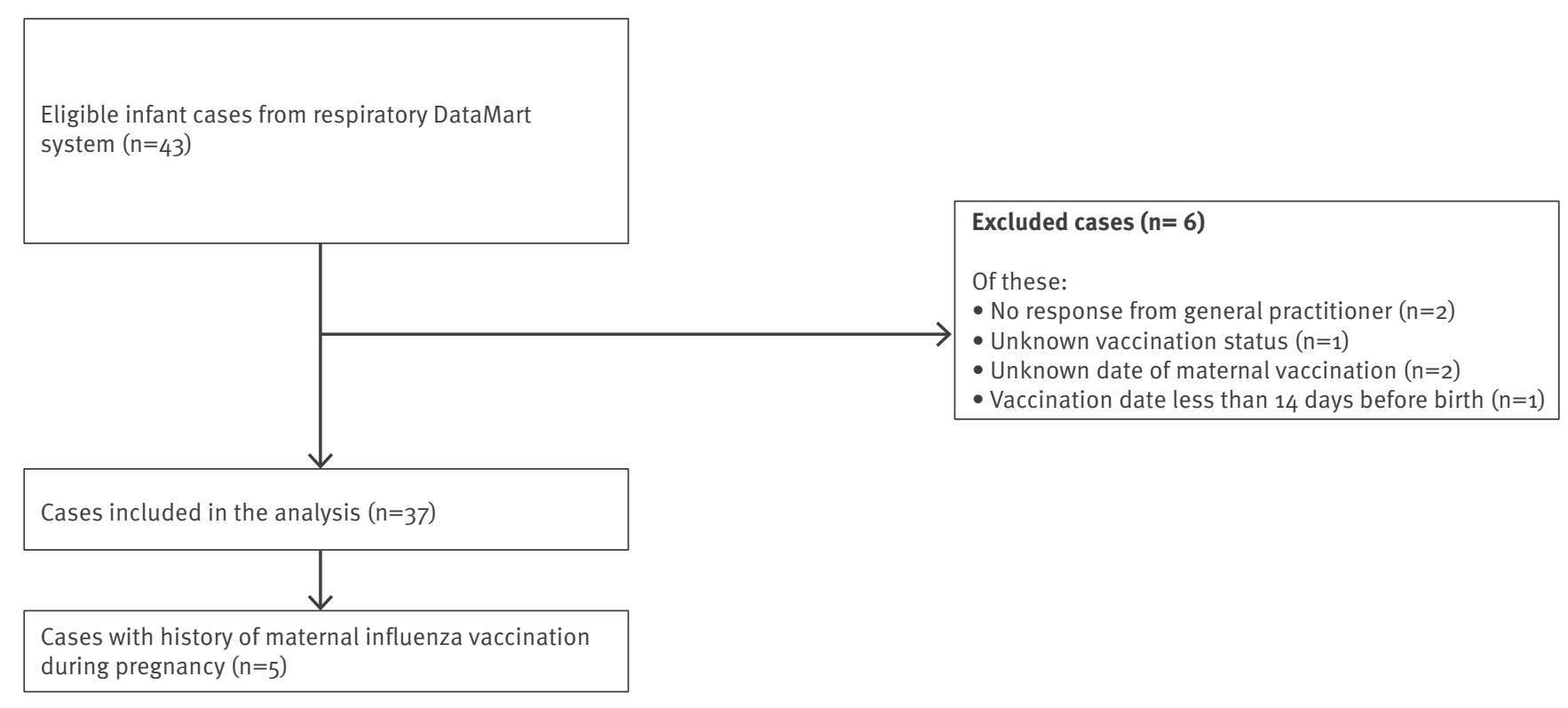

transfer from mother to foetus but also a likely indirect effect from preventing influenza infection in mothers and subsequent secondary transmission to infants.

A strength of these findings, besides being the first such estimates in Europe in any season, also represent the first VE estimates for seasonal influenza vaccination during pregnancy in the $2013 / 14$ season. In addition, to our knowledge, this is the first study to use the screening method to specifically estimate the effectiveness of vaccination during pregnancy to prevent infant influenza infection. The advantage of this approach is that routinely collected vaccine coverage and case-level data can be adjusted for key confounders to rapidly estimate and disseminate VE prior to the next influenza season. This importantly facilitates comparison of VE between different seasons as circulating influenza subtypes vary between influenza seasons and thus in the closeness of matching to the annual seasonal vaccine. In our study, the influenza subtypes identified among cases closely resembled circulating influenza in the wider population, with the 2009 pandemic influenza $A\left(\mathrm{H}_{1} \mathrm{~N}_{1}\right)$ virus predominating in 2013/14 [16].

A strength of this approach is the use of laboratoryconfirmed endpoints to provide a more specific VE estimate compared to influenza-like Illness. One potential limitation is the relatively low intensity season seen in $2013 / 14$, which resulted in relatively small numbers of cases in the study and wide Cls. Despite this, evidence of significant effectiveness was observed, although not the ability to examine sub-type specific protection. A further potential limitation of the screening method is that VE can only be adjusted for covariates measured in the population vaccine coverage data; although we were able to adjust for factors such as month, we were not able to examine the effects of trimester of vaccination, prior maternal vaccination or breastfeeding in this study. Furthermore, if unvaccinated mothers took ill infants to health services less frequently than vaccinated mothers, this may bias the VE towards a lower estimate; however such a potential bias due to differences in healthcare seeking would be less important for severe illness requiring hospitalisation. The cases included in this study represent those tested for clinical purposes. Such a selection could potentially bias VE estimates, if the decision to test was associated with the vaccination status of infants' mothers; however such an effect would be limited as clinicians are unlikely to have been aware of mothers' vaccination status.

A significant burden of influenza illness is observed in young infants when compared to children and young adults. Preventive measures are needed to reduce influenza-related morbidity among infants, and possibly alleviate pressures on health services, including primary and secondary care. Our study results suggest that seasonal influenza vaccination during pregnancy is effective in preventing laboratory-confirmed influenza illness among infants, potentially helping to address this burden of illness. Although further such studies are needed, particularly in Europe, vaccine uptake in pregnant women can be further improved and our findings can be used to support pregnant women to make informed decisions about seasonal influenza vaccination in pregnancy. 


\section{Acknowledgements}

We would like to thank the laboratories participating in the Respiratory DataMart System (RDMS) and the general practitioners who responded with vaccination information, for their assistance. We are also grateful to the reviewer for their comments.

This work was undertaken as a routine public health function to monitor vaccination programmes; PHE holds permissions to collect data under Section 251 of the National Health Service Act 2006 and the 2002 Health Service (Control of Patient Information) regulations as part of monitoring the performance of the national vaccination programme.

\section{Conflicts of interest}

All authors employed by PHE which is responsible for monitoring of influenza immunisation programmes. MZ reports as a government body representative, frequent interactions with colleagues in vaccine industry to ensure good publicprivate liaison activities for national vaccine campaigns. MD reports personal fees from Sanofi Pasteur MSD, non-financial support from Janssen, non-financial support from the European Centre for Disease Prevention and Control (ECDC), outside the submitted work; and is an employee of public health England (PHE). PHE is a government agency that provides guidance on multiple aspects of influenza management, treatment, prevention and public health.

\section{Authors' contributions}

All authors contributed to the design of the project and writing and editing of the manuscript.

Data Collection: GD, HZ, FB

Data Analysis: GD, NA, RP

GD undertook the data analysis and had full access to all of the data in the study and takes responsibility for the integrity of the data and the accuracy of the data analysis.

\section{References}

1. Neuzil KM, Mellen BG, Wright PF, Mitchel EF Jr, Griffin MR. The effect of influenza on hospitalizations, outpatient visits, and courses of antibiotics in children. N Engl f Med. 2000;342(4):225-31. http://dx.doi.org/10.1056/ NEJM200001273420401 PMID:10648763

2. Cromer D, van Hoek AJ, Jit M, Edmunds WJ, Fleming D, Miller $E$. The burden of influenza in England by age and clinical risk group: a statistical analysis to inform vaccine policy. J Infect. 2014;68(4):363-71. http://dx.doi.org/10.1016/j.jinf.2013.11.013 PMID:24291062

3. Campbell CNJ, Mytton OT, McLean EM, Rutter PD, Pebody RG, Sachedina N, et al. Hospitalization in two waves of pandemic influenza $A\left(H_{1} N_{1}\right)$ in England. Epidemiol Infect. 2011;139(10):1560-9. http://dx.doi.org/10.1017/ So950268810002657 PMID:21108872

4. Public Health England (PHE). Immunisation against infectious diseases. Guidance Influenza: the green book, chapter 19. London: PHE. [Accessed 14 Nov 2013]. Available from: https://www.gov.uk/government/publications/ influenza-the-green-book-chapter-19

5. Puleston R, Bugg G, Hoschler K, Konje J, Thornton J, Stephenson I, et al. Multi-centre observational study of transplacental transmission of influenza antibodies following vaccination with $\mathrm{ASO}_{3}(\mathrm{~A})$-adjuvanted $\mathrm{H}_{1} \mathrm{~N}_{1} 2009$ vaccine. PLoS ONE. 2013;8(1):e47448. http://dx.doi.org/10.1371/journal. pone.0047448 PMID:23372640

6. Centers for Disease Control and Prevention (CDC). Prevention and control of seasonal influenza with vaccines. Recommendations of the Advisory Committee on Immunization Practices--United States, 2013-2014. MMWR Recomm Rep. 2013;62(RR-07):1-43. PMID:24048214

7. Public Health Agency of Canada (PHAC). National Advisory Committee on Immunization (NACl). Statement on Seasonal Influenza Vaccine for 2013-2014.Ontario: PHAC. [Accessed 24 Jul 2014]. Available from: http://www. phac-aspc.gc.ca/ publicat/ccdr-rmtc/13vol39/acs-dcc-4/index-eng.php\#rec
8. Zaman K, Roy E, Arifeen SE, Rahman M, Raqib R, Wilson E, et al. Effectiveness of maternal influenza immunization in mothers and infants. N Engl J Med. 2008;359(15):1555-64. http://dx.doi.org/10.1056/NEJMoa0708630 PMID:18799552

9. Poehling KA, Szilagyi PG, Staat MA, Snively BM, Payne DC, Bridges CB, et al.; New Vaccine Surveillance Network. Impact of maternal immunization on influenza hospitalizations in infants. Am J Obstet Gynecol. 2011;204(6) Suppl 1;S141-8. http://dx.doi.org/10.1016/j.ajog.2011.02.042 PMID:21492825

10. Benowitz I, Esposito DB, Gracey KD, Shapiro ED, Vázquez $M$. Influenza vaccine given to pregnant women reduces hospitalization due to influenza in their infants. Clin Infect Dis. 2010;51(12):1355-61. http://dx.doi.org/10.1086/657309 PMID:21058908

11. Zhao H, Green H, Lackenby A, Donati M, Ellis J, Thompson C, et al. A new laboratory-based surveillance system (Respiratory DataMart System) for influenza and other respiratory viruses in England: results and experience from 2009 to 2012. Euro Surveill. 2014;19(3):20680. http://dx.doi.org/10.2807/15607917.ES2014.19.3.20680 PMID:24480060

12. Public Health England (PHE). Immform. London: PHE. [Accessed 24 Jul 2014]. Available from: https://www.gov.uk/government/ collections/immform

13. Farrington CP. Estimation of vaccine effectiveness using the screening method. Int J Epidemiol. 1993;22(4):742-6. http:// dx.doi.org/10.1093/ije/22.4.742 PMID:8225751

14. Widgren K, Magnusson M, Hagstam P, Widerström M, Örtqvist $\AA$, Einemo IM, et al. Prevailing effectiveness of the 2009 influenza $A\left(\mathrm{H}_{1} \mathrm{~N}_{1}\right)$ pdmog vaccine during the $2010 / 11$ season in Sweden. Euro Surveill. 2013;18(15):20447. PMID:23594578

15. Thomas HL, Andrews N, Green HK, Boddington NL, Zhao H, Reynolds A, et al. Estimating vaccine effectiveness against severe influenza in England and Scotland 2011/2012: applying the screening method to data from intensive care surveillance systems. Epidemiol Infect. 2014;142(1):126-33. PMID:23591102

16. Public Health England (PHE). Surveillance of influenza and other respiratory viruses in the United Kingdom: Winter 2013/14. London: PHE. Jun 2014. Available from: http:// www.hpa.org.uk/webw/HPAweb\&HPAwebStandard/ HPAweb_C/1317141330709 